\title{
Measurement and Simulation of One-Dimensional Transient Three-Phase Flow for Monotonic Liquid Drainage
}

\author{
R. J. LENHARD \\ Virginia Polytechnic Institute and State University, Blacksburg \\ J. H. DANE \\ Auburn University, Auburn, Alabama \\ J. C. Parker and J. J. Kaluarachchi \\ Virginia Polytechnic Institute and State University, Blacksburg
}

\begin{abstract}
Simultaneous movement of oil, water, and air in a sandy porous medium was investigated experimentaliy under transient flow conditions and results were compared to numerical simulations employing a finite element multiphase flow code. The liquid hydrocarbon was Soltrol 170, a low-density branched alkane mixture. Liquid saturations were measured using a collinear dual-energy gamma radiation apparatus and liquid pressures were measured using hydrophilic (untreated) and hydrophobic (treated) ceramic tensiometers connected to pressure transducers. The experimental regime was selected to impose monotonically draining water and total liquid saturation paths to avoid hysteretic effects. Measured saturations and pressures are compared to values obtained from numerical simulations of the experiment using a finite element solution of the governing multiphase flow equations assuming negligible gas pressure gradients. Functional relationships between permeabilities $k$, saturations $S$, and capillary pressures $P$ employed in the numerical model were estimated by two calibration methods which require different degrees of experimental effort. Measured transient water saturation versus oil-water capillary head data agreed well with predictions from static air-water $S-P$ relations and interfacial tension data. Transient total liquid saturation versus air-oil capillary head data deviated more severely from the scaled air-water S-P data, possibly reflecting noncompliance with the assumption of negligible gas pressure gradients. Reasonably good agreement was observed between measured and numerically simulated water and oil saturations and pressures in space and time. Sensitivity of the numerical results to calibration method was not great.
\end{abstract}

\section{INTRODUCTION}

Historically, investigations of three-phase fluid flow have been conducted largely within the petroleum industry. However, since the movement of nonaqueous phase liquids (NAPL) within the vadose zone is of major environmental concern, interest has grown recently in the water resources arena in the physics of air-NAPL-water flow phenomena and the development of models for subsurface NAPL movement. A number of numerical codes based on finite difference [Abriola and Pinder, 1985; Faust, 1985; Baehr and Corapcioglu, 1987] and finite element [Hochmuth and Sunada, 1985; Osborne and Sykes, 1986; Kuppusamy et al., 1987] schemes have been developed to solve the system of highly nonlinear partial differential equations governing multiphase flow in groundwater and vadose zones.

Utilization of such models requires accurate representations of functional relations between fluid permeabilities $k$, saturations $S$, and capillary pressures $P$. Typically, $k-S-P$ relations for two-fluid phase systems (i.e., air-NAPL and NAPL-water) are extended to predict fluid behavior in three-phase airNAPL-water systems [Aziz and Settari, 1979]. Extending twofluid phase $S-P$ relations to three-fluid phase systems is commonly employed because of the experimental difficulty of di-

Copyright 1988 by the American Geophysical Union.

Paper number 7 W4969. $0043-1397 / 88 / 007 \mathrm{~W}-4969 \$ 05.00$ rectly measuring $k-S-P$ relations in three-fluid phase porous media systems. The basis for extending two-phase air-NAPL and NAPL-water relations to three-phase air-NAPL-water systems, when fluid wettabilities follow the order water to NAPL to air, originates from theoretical and experimental work of Leverett [1941], Leverett and Lewis [1941], Corey et al. [1956], and others. Recently, Lenhard and Parker [1988] developed an experimental cell capable of directly measuring air-NAPL-water $S-P$ relations in unconsolidated porous media and validated the assumptions of extending two-phase air-NAPL and NAPL-water $S-P$ relations to air-NAPL-water fluid systems for monotonically draining liquid saturation paths in sandy porous media.

Parker et al. [1987] utilized these assumptions to develop a parametric model describing $k-S-P$ relations of three-phase air-NAPL-water systems for monotonically draining water and total liquid saturation paths in water-wet porous media systems. In this model, two-phase air-water, air-NAPL, and NAPL-water $S-P$ relations were scaled to obtain a unique function for a given porous medium by applying linear transformations to the capillary heads such that

$$
\begin{aligned}
\bar{S}_{w}{ }^{a w}\left(\beta_{a w} h_{a w}\right) & =S^{*}\left(h^{*}\right) \\
\bar{S}_{o}{ }^{a o}\left(\beta_{a o} h_{a a}\right) & =S^{*}\left(h^{*}\right) \\
S_{w}{ }^{o w}\left(\beta_{o w} h_{o w}\right) & =S^{*}\left(h^{*}\right)
\end{aligned}
$$

where $S^{*}\left(h^{*}\right)$ is the scaled saturation capillary head function; $\beta_{i j}$ are scaling coefficients for fluid pair $i j$ ( $i$ denotes the non- 
wetting phase, $j$ the wetting phase, and $i, j=a, 0, w$ indicate air, oil, and water phases, respectively, where "oil" and "NAPL" are hereafter used interchangeably); and $\bar{S}_{j}{ }^{i j}$ is the effective wetting phase saturation of two fluid phase system $i j$ and $h_{i j}$ is the ij pair capillary head defined as

$$
\begin{gathered}
\bar{S}_{j}{ }^{i j}=\left(S_{j}{ }^{i j}-S_{m}\right) /\left(1-S_{m}\right) \\
h_{i j}=\left(P_{i}-P_{j}\right) / \rho^{0} g
\end{gathered}
$$

respectively. Where $S_{j}{ }^{j}$ is the actual wetting phase saturation in the two-fluid phase system, $S_{m}$ is an "irreducible" wetting phase saturation, $P_{i}$ and $P_{j}$ are pressures in fluids $i$ and $j, \rho^{0}$ is a reference liquid density taken equal to that of pure water, and $g$ is gravitational acceleration. Since the scaling is based upon an arbitrary reference system, we set $\beta_{a w}=1$.

Saturation-pressure relations in three-fluid phase water-wet systems are assumed to be described by

$$
\begin{aligned}
S_{w}\left(\beta_{o w} h_{o w}\right) & =S^{*}\left(h^{*}\right) \\
S_{x}\left(\beta_{a o} h_{a o}\right) & =S^{*}\left(h^{*}\right)
\end{aligned}
$$

where $\bar{S}_{w}$ and $\bar{S}_{\mathrm{r}}$ are effective water and total liquid (i.e., water and NAPL) saturations in the three-phase system defined in the same manner as for the two-phase system (equation (2)) The $S^{*}\left(h^{*}\right)$ function is assumed to be of the form employed by van Genuchten [1980]

$$
S^{*}\left(h^{*}\right)=\left[1+\left(\alpha h^{*}\right)^{n}\right]^{-1+1 / n}
$$

where $\alpha$ and $n$ are curve shape parameters. Closed-form expressions for two- and three-phase relative permeabilitysaturation relations may be derived by employing $S^{*}\left(h^{*}\right)$ as an index of the pore size distribution [Parker et al., 1987]. Numerical implementation of the $k-S-P$ model in a multiphase finite element code and experimental validation for two-phase NAPL-water flow have been reported by Kuppusamy et al. [1987].

Relatively little experimental research has been conducted to elucidate fluid-porous medium properties governing threefluid phase flow in the vadose zone. Eckberg and Sunada [1984] studied infiltration of oil into partially saturated porous media and concluded that water saturation at static equilibrium in a three-phase air-oil-water system was a function solely of oil-water capillary head. They also observed that total liquid content was apparently not a single-valued function of the air-oil capillary pressure, contrary to assumptions proposed by Leverett [1941] and validated by Lenhard and Parker [1988]. However, possible complications due to hysteresis were not considered [Parker and Lenhard, 1987; Lenhard and Parker, 1987b] and direct measurements of oil pressures were unavailable.

Schiegg [1980] employed hydrophilic and hydrophobic pressure probes and a gamma radiation apparatus to measure oil propagation in a two-dimensional experimental flume containing quartz sand. His objective was to minimize experimental error in measuring oil and water saturations and pressures during different oil contamination scenarios to enable validation of numerical models of multiphase flow. A partial summary of Schiegg's work is given by Schiegg and McBride [1988]. To our knowledge, Schiegg's data have as yet not been employed for numerical model validation.

Experimental work dealing with three-phase fluid flow has also been conducted by researchers in the petroleum industry. Petroleum researchers have developed a variety of methods for determining three-phase $k-S$ relations [Honarpour et al. 1986] and for direct measurement of oil and water saturations in three-phase fluid systems [Snell, 1962; Saraf and Fatt, 1967; Oak and Ehrlich, 1985], but have apparently not given much attention to the problem of measuring three-phase $S-P$ relations.

The purpose of the present paper is to discuss experimental procedures for simultaneously measuring fluid saturations and pressures of three-fluid phase porous media systems in the laboratory and to compare measured liquid saturations and pressures in a transient three-phase flow experiment with numerical simulations based on the $k-S-P$ model of Parker et al. [1987]. Water and NAPL saturations were determined with a dual-energy gamma radiation apparatus and liquid pressures were measured using hydrophobic and hydrophilic ceramic tensiometers. The experimental regime imposed monotonically draining water and total liquid saturation paths to avoid hysteretic effects to evaluate the $k-S-P$ model under conditions for which it should be valid.

\section{Materials AND Methods}

\section{Fluid and Porous Medium Properties}

The porous medium employed in this study was an unconsolidated sandy material comprised of $4.8,94.7,0.4$, and $0.3 \%$ fine gravel, sand, silt, and clay sizes (USDA), respectively. The average bulk density of the sand packed in the flow column as measured with the dual-energy gamma radiation apparatus was $1.60 \mathrm{~g} \mathrm{~cm}^{-3}$ which corresponds to a porosity of 0.40 . The NAPL was a 1:9 mixture by volume of 1 iodoheptane and Soltrol 170, a hydrocarbon solvent produced by Phillips Chemical Company consisting of a mixture of branched alkanes with carbon numbers ranging from $\mathrm{C} 10$ to C16 and having very low solubility in water. The aqueous phase was a $7 \%$ solution of $\mathrm{NaBr}$ by weight. Addition of $\mathrm{NaBr}$ and 1-iodoheptane to water and Soltrol, respectively, was employed to enhance differences in gamma attenuation coeflicients [Oak and Ehrlich, 1985]. The aqueous phase and NAPL mixture densities were 1.05 and $0.83 \mathrm{~g} \mathrm{~cm}^{-3}$, respectively. Air-water, air-oil, and oil-water interfacial tensions were measured by the drop method [Adamson, 1967] in quintuplicate using the mixtures after they were in contact with the sandy porous medium for an extended period (in excess of 90 hours) yielding values of $73.8,26.0$ and $43.3 \mathrm{mN} \mathrm{m}^{-1}$, respectively. The drop method is an old fairly accurate technique which is based on the premise that the maximum force available to support the weight of a liquid droplet is related to the interfacial tension force $\left(F \mathrm{~L}^{-1}\right)$ times the circumference of the tip of a capillary tube from which the liquid is displaced [Adamson, 1967]. Liquids were mixed with the porous medium before interfacial tension measurements were conducted to obtain values reflective of those in the porous medium.

To calibrate the $k-S-P$ model employed in the multiphase flow code, two-phase air-water $S-P$ relations were measured in triplicate according to procedures outlined by Lenhard and Parker [1987a] on $5.26 \mathrm{~cm}$ diameter $\times 5.18 \mathrm{~cm}$ length cores of the sandy porous medium packed to the same average bulk density measured in the transient flow experiment. Details pertaining to calibration of the $S-P$ model will be discussed in the section on numerical simulation. Saturated conductivities were measured by a falling head method on triplicate cores having the same dimensions used for air-water $S-P$ analyses. Repli- 


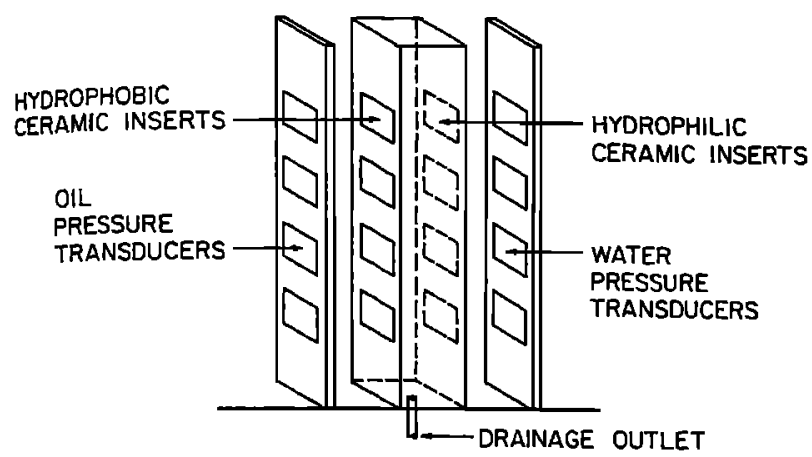

Fig. 1. Experimental three-phase flow column design for simultaneous measurement of oil and water pressures.

cate means of water and oil saturated conductivities were 77.4 and $37.7 \mathrm{~cm} \mathrm{~h}^{-1}$, respectively, using fluid pressure heads defined in water equivalent heights [Kuppusamy et al., 1987].

\section{Flow Cell Design and Liquid Pressure Measurements}

A Plexiglas flow cell (Figure 1), $100 \mathrm{~cm}$ in height, and $7.5 \mathrm{~cm}$ square in cross section was equipped with specially designed porous ceramic tensiometers to enable independent measurement of NAPL and water phase pressures. Soltrol does not interact with Plexiglas in a manner to prohibit its use and the duration of the experiment was not long enough to permit spreading of the oil along the Plexiglas walls due to wettability effects [see Schiegg and McBride, 1988]. Ceramic tensiometers connected to pressure transducers (Figure 1) were placed at $10 \mathrm{~cm}$ intervals on both sides of the cell with water and NAPL phase pressures measured on opposite sides at 5 elevations. Drainage of liquid from the column was accommodated by an outlet at the base of the cell which was large enough not to restrict liquid outflow during drainage.

Measurements of liquid phase pressures were accomplished by establishing continuous liquid phases between the porous medium and pressure transducers located outside the flow cell. Continuous water and oil phases were obtained by employing untreated and treated ceramic tensiometers, respectively. When fluid wettabilities decrease in the order water to oil to air, a continuous water phase will extend from pores in the soil to those in untreated ceramic plates provided the oilwater capillary head does not exceed the oil-entry capillary head of the ceramic. The latter is a function of the largest continuous pore size in the ceramic and the interfacial tension between oil and water. A continuous oil phase may be obtained by treating the ceramic to make it hydrophobic. This was achieved with chlorotrimethylsilane, which forms durable $\mathrm{Si}-\mathrm{O}-\mathrm{Si}$ bonds with the ceramic surface, while attached trimethyl groups repell water molecules in preference to oil and air [Ogden, 1985]. Ceramic sections were immersed in chlorotrimethylsilane for approximately 2 hours and then rinsed thoroughly with toluene followed by methanol. Caution needs to be exercised when working with chlorotrimethylsilane because it may react violently with water.

\section{Simultaneous Determination of Water and Oil Saturations}

The Auburn University collinear dual-energy (Am 241 and Cs 137) gamma radiation system, as described by Hopmans and Dane [1985, 1986], was used to simultaneously determine volumetric fluid contents of a three-phase gas-NAPL-water porous medium system. Measurements were conducted at the elevations of the ceramic tensiometers. This was accomplished by moving two interconnected parallel platforms, one supporting the collinear radiation sources and the other supporting the detector, to the five specified locations with stepper motors under computer control. By employing stepper motors, the parallel platforms can be returned repeatedly to the same measurement location. Both the collinear sources and the detector possessed lead shielding with $6 \mathrm{~mm}$ diameter collimators.

The general attenuation equation for a radioactive beam passing through a porous medium containing three fluids, namely, water $\left(u^{\prime}\right)$, NAPL $(n)$, and gas $(g)$ can be derived from Beer's law as

$$
I=I_{0} \exp \left(-\mu_{s} \rho_{s} x-\mu_{w} \rho_{w} \theta_{w} x-\mu_{n} \rho_{n} \theta_{n} x-\mu_{g} \rho_{\theta} \theta_{\theta} x\right)
$$

in which $I$ is the exiting radiation intensity expressed as a count rate (counts per second (cps)); $l_{0}$ is the incident count rate (cps); $\mu_{i}$ are mass attenuation coefficients $\left(\mathrm{cm}^{2} \mathrm{~g}^{-1}\right) ; \rho_{i}$ are mass densities $\left(\mathrm{g} \mathrm{cm}^{-3}\right) ; \theta_{i}$ are volumetric fluid contents $\left(\mathrm{cm}^{3} \mathrm{~cm}^{-3}\right)$; and $x$ is the path length through the porous medium (cm); where $i$ denotes soil $(s)$, water $(w)$, NAPL $(n)$, or gas $(g)$. Assuming the mass density of soil $\rho_{s}$, i.e., the bulk density of the porous medium, to be time invarient and the contribution of the gas term to be negligible, (6) can be rewritten as

$$
I=I_{0}^{\prime} \exp \left(-\mu_{w} \rho_{w} \theta_{w} x-\mu_{n} \rho_{n} \theta_{n} x\right)
$$

where $I_{0}^{\prime}=I_{0} \exp \left(-\mu_{\mathrm{s}} \rho_{\mathrm{s}} x\right)$.

Following Nofziger and Swartzendruber [1974] and differentiating between energy sources Am 241 (subscript a) and Cs 137 (subscript $c$ ), (7) yields

$$
\begin{aligned}
& I_{a}=I_{0 a}{ }^{\prime} \exp \left(-U_{w a} \theta_{w}-U_{n a} \theta_{n}\right) \\
& I_{c}=I_{0 c}{ }^{\prime} \exp \left(-U_{w c} \theta_{w}-U_{n c} \theta_{n}\right)
\end{aligned}
$$

where $U_{i j}=\mu_{i j} \rho_{i} x$ in which subscripts $i$ and $j$ indicate the material and energy source, respectively, and

$$
I_{0 j}^{\prime}=I_{0 j} \exp \left(-\mu_{s j} \rho_{s} x\right)
$$

Solving ( $8 a)$ and $(8 b)$ for $\theta_{w}$ and $\theta_{n}$ yields

$$
\begin{aligned}
& \theta_{w}=\left[U_{n a} \ln \left(I_{0 c}^{\prime} / I_{c}\right)-U_{n c} \ln \left(I_{0 a}^{\prime} / I_{a}\right)\right] / k \\
& \theta_{n}=\left[U_{w c} \ln \left(I_{0 a}^{\prime} / I_{a}\right)-U_{w a} \ln \left(I_{0 c}^{\prime} / I_{c}\right)\right] / k
\end{aligned}
$$

where $k=U_{w c} U_{n a}-U_{w a} U_{n c}$. To solve (9), $U_{n a}, U_{n e}, U_{w a}, U_{w c}$, $I_{0 a}{ }^{\prime}$, and $I_{o c}{ }^{\prime}$ must be know. Note that now the subscript $i$ in $U_{i j}=\mu_{i j} \rho_{i}$ terms refers only to water and NAPL since gamma attenuation associated with a gas phase is assumed to be negligible and $\mu_{s j}$ and $\rho_{s}$ terms are contained in $I_{o j}$.

Determination of $\rho_{i}, \mu_{w j}, \mu_{n j}$, and $\mu_{s j}$ were conducted in preliminary experiments employing a small cubical Plexiglas cell of similar cross section as the experimental flow cell which was divided into four parallel vertical compartments. Gamma count rates were obtained first on the empty cell to obtain $I_{0 j}$ and then on the cell after the compartments were sequentially filled with the material of concern, i.e., water, NAPL, or soil to obtain $I_{j}$ corresponding to the number of filled compartments. The cell was positioned in the gamma beam such that the radiation traversed through all compartments, and the geometry between sources, detector, and compartmental cell was held constant for all measurements. This procedure was replicated 4 times resulting in 20 measurements of gamma radiation attenuation as a function of path length for each material. 
The product $\mu_{i j} \rho_{i}$ for water and NAPL could be determined by regression of the 20 data pairs to the linearized attenuation equation

$$
\ln \left(I_{j}\right)=\ln \left(I_{\mathrm{oj}}\right)-\mu_{i j} p_{i} y
$$

where $y$ is the path length through the filled compartments, i.e.,

$$
y=\sum_{k=1}^{n} y_{k}
$$

in which $n$ is the number of filled compartments and $y_{k}$ is the path length through compartment $k$. Values for $y_{k}(k=1-4)$ were obtained from caliper measurements. Note that in (10) $\theta_{w}$ or $\theta_{n}$ does not appear as in (6). This is because the calibration technique for the aqueous phase and for the NAPL uses liquids only, i.e., $\theta_{i}=1$, and not mixtures of porous media and liquids [e.g., Ferrand et al., 1986] which reduces the time required for calibration and eliminates experimental errors in determining $\theta$, for calibration purposes.

Since filling the compartmental cell with soil (air dry) inevitably results in somewhat different soil and water mass densities in each compartment, (10) could not be employed for determination of $\mu_{s j}$. To determine the mass attenuation of soil $\left(\mu_{s j}\right.$ when $\left.\theta_{w}=0\right)$, the composite soil and water mass densities in the gamma radiation path need to be considered. Therefore $\mu_{s j}$ are determined by regression to

$$
\ln \left(I_{j}\right)+\mu_{w j} \rho_{w} \theta_{w}^{\prime}=\ln \left(I_{0 j}\right)-\mu_{s j} \rho_{s}^{\prime} y
$$

where $\theta_{w}{ }^{\prime}$ is the composite volumetric water content, $\rho_{s}^{\prime}$ is the composite soil bulk density, and the other terms have been defined previously. Volumetric water content and soil bulk density for each compartment were determined gravimetrically. Values for $\mu_{w a} \rho_{w a}, \mu_{w c} \rho_{w c}, \mu_{n a} \rho_{n a}, \mu_{n c} \rho_{n c}, \mu_{s a}$, and $\mu_{s c}$ so obtained were $0.337596,0.088411,0.735462,0.071888$, $\mathrm{cm}^{-1}$ and 0.242413 and $0.076085 \mathrm{~cm}^{2} \mathrm{~g}^{-1}$, respectively.

To complete the determinations of $U_{i j}$ and $I_{0 j}$ as needed for (9), values for the path lengths $x$ at the 5 positions along the flow column were required. They were obtained by first recording count rates when the flow column was empty resulting in a $I_{0 j}$ measurement for each location. Next the flow column was filled with a liquid of known $\mu_{i j} \rho_{i}$, i.e., water, and count rates were recorded resulting in a measured $I_{j}$ for each of the elevations. Path lengths corresponding to each measurement position along the flow column could then be calculated from

$$
x=\ln \left(I_{0 j} / I_{j}\right) / \mu_{w j} \rho_{w}
$$

The remaining two parameters needed for (9) are $I_{0 a}{ }^{\prime}$ and $I_{0 c}{ }^{\prime}$. These values, according to $(8 c)$, can only be obtained if the bulk density of the soil is known at each of the five locations (note $I_{0 c}$ and $I_{0 c}$ were previously measured during determination of path lengths). After packing and saturating the porous medium in the flow cell with water, gamma count measurements $I_{j}$ were obtained at all five positions to simultaneously determine the initial water content and bulk density from known $I_{0 j}, I_{j}$, and $U_{i j}$ values [Nofziger and Swartzendruber, 1974; Hopmans and Dane, 1986]. Count rates measured at the five elevations along the flow column were obtained by sequentially moving radiation sources and detector from positions 1 through 5. This procedure was repeated 4 times. By employing this calibration procedure we characterized $U_{i j}$ and $I_{0 j}{ }^{\prime}$ at each measurement location, thereby accounting for dif- ferences in path length along an experimental flow cell contrary to the procedure outlined by Ferrand at al. [1986] in which path length is assumed to be constant.

During the calibration procedure all counting times were 5 min in duration. Path lengths $x$ calculated for Am 241 and $C_{s}$ 137 using (13) differed by a maximum of $1.17 \%$. Volumetric water and oil contents for the transient flow experiment based on these path lengths differed by less than $1 \%$. This discrepancy in path lengths was considered to be acceptable and mean values were employed for all calculations of the transient flow experiment.

All count rates were corrected for resolving time as outlined by Fritton [1969]. Radiation emitted by the Cs 137 source, but detected in the Am 241 window, was accounted for by the method of Nofziger and Swartzendruber [1974]. All count rates in the above equations refer therefore to corrected count rates.

With all parameters known in (9), $\theta_{w}$ and $\theta_{n}$ could be determined during transient conditions. Volumetric fluid contents were converted into fluid saturations using measured soil bulk density to calculate the porosity at each of the five measurement positions (i.e., saturation equals volumetric fluid content/porosity). During the transient flow experiment gamma radiation count rates were obtained over $1 \mathrm{~min}$ periods.

\section{Imposed Experimental Initial and Boundry Conditions}

The sandy porous medium was packed on top of a 4-cmthick coarse gravel layer to a height of $56.5 \mathrm{~cm}$ from the bottom of the flow column. The gravel layer ensured that drainage of liquid from the sand column would not be impeded as water exited from the bottom of the flow cell. The packed column was gently flushed with $\mathrm{CO}_{2}$ before imbibing water slowly through the bottom of the cell to minimize gas entrapment. In addition, the water saturated column was left undisturbed for approximately 12 hours to enable entrapped gas to dissolve in the pore fluid. Locations of the ceramic tensiometers (i.e., measurement locations) were at 5.2, 15.2, $25.2,35.2$, and $45.2 \mathrm{~cm}$ below the soil surface.

Before drainage of liquid from the column commenced, 200 $\mathrm{mL}$ of the NAPL mixture was ponded on top of the water saturated soil. The initial depth of oil on the soil surface was $3.56 \mathrm{~cm}$. Drainage of liquid from the column at $t=0$ was permitted after the outflow elevation was positioned at 25.2 $\mathrm{cm}$ below the soil surface. This was accomplished by lowering the outlet of a flexible hose which was connected to the bottom of the flow cell from its initial position (level with the upper soil surface, $z=0$ ). Measurements of liquid saturations and pressures were initiated shortly after drainage commenced. The outflow point was reset to $1.5 \mathrm{~cm}$ above the lower soil boundary at $t=41.5 \mathrm{~min}$ to allow additional drainage. This stepwise drainage procedure was used to prevent excessively large flow rates during the time period employed for gamma counts. The imposed initial and boundary conditions ensured monotonically draining water and total liquid saturation paths.

\section{Numerical Simulation}

A finite element code which solves the coupled partial differential equations governing flow in a three-fluid phase porous medium system with assumed negligible gas pressure gradients was used to simulate the experiment. Basic model formulation is similar to that described by Kuppusamy et al. [1987] but with variable upstream weighting of spatial terms to improve 
TABLE 1. Parameters of the Three-Phase $k-S$-P Model Employed in Numerical Simulations as Obtained by Two Calibration Methods

\begin{tabular}{lcc}
\hline Parameters & Method 1 & Method 2 \\
\hline$\alpha \beta_{u m}, \mathrm{~cm}^{-1}$ & 0.054 & $\ldots$ \\
$\alpha \beta_{a u}, \mathrm{~cm}^{-1}$ & 0.154 & 0.104 \\
$\alpha \beta_{u, \ldots}, \mathrm{cm}^{-1}$ & 0.092 & 0.091 \\
$n$ & 3.248 & 2.729 \\
$S_{m}$ & 0.0 & 0.0 \\
$\phi$ & 0.40 & 0.40 \\
$K_{s ! n}, \mathrm{~cm} \mathrm{~h}^{-1}$ & 77.4 & 77.4 \\
$K_{s o}, \mathrm{~cm} \mathrm{~h}^{-1}$ & 37.7 & 37.7 \\
\hline
\end{tabular}

accuracy and robustness, and with refinements in the evaluation of time derivatives terms to improve convergence and mass balance characteristics of the solution (J. J. Kaluarachchi and J. C. Parker, Improving the efficiency of finite element methods for modeling multiphase flow, submitted to Water Resources Research, 1988). The $k-S-P$ model of Parker et al. [1987] was employed in the numerical code. Parameters in the model consist of $\alpha, n, S_{m}, \beta_{a w}, \beta_{a o}$, and $\beta_{o w}$ as defined by (1)-(5), porosity $\Phi$, and water and oil saturated conductivities, $K_{s w}$ and $K_{s o}$, respectively. Values of $\Phi, K_{s w}$ and $K_{s o}$ were determined as previously discussed. Due to the coarse grain size of the porous medium, we assumed $S_{m}=0$ [see Lenhard and Parker, 1987a]. Since contiguous air and water phases are absent in the experiment considered here, the factor $\alpha \beta_{a w}$ is not required for the simulations. The remaining parameters needed to calibrate the model are $n, \alpha \beta_{a o}$ and $\alpha \beta_{o w}$ (note parameters $\alpha$ and $\beta_{i j}$ occur only as products in the constitutive equations).

Two methods were utilized to evaluate the latter parameters. In method 1 , independently measured air-water $S$ - $P$ data were used to fit parameters $n$ and $\alpha$ (the air-water system was arbitrarily taken as the scaling reference, i.e., $\beta_{a w}=1$ ), and scaling factors $\beta_{a o}$ and $\beta_{o w}$ were obtained from ratios of interfacial tensions as described by Lenhard and Parker [1987a]. In method 2, the parameters $n, \alpha \beta_{a 0}$, and $\alpha \beta_{o w}$ were obtained by fitting water saturation versus oil-water capillary head data and total liquid saturation versus air-oil capillary head data measured during the transient flow experiment to (4) and (5). Fluid saturations were determined with the dual-energy gamma apparatus and capillary heads were obtained from the oil and water pressure measurements. Air pressure was assumed to be constant and equal to that of the ambient atmosphere. Values of the parameters employed in the simulations for both calibration methods are listed in Table 1 .

For convenience of discussion, we define a vertical coordinate $z$ with reference $(z=0)$ at the upper soil surface and measured positive downwards. Initial water and oil pressure heads at commencement of liquid drainage $(t=0)$ were described by the equilibrium conditions

$$
\begin{gathered}
h_{w}(z, 0)-z=3.56\left(\rho_{o} / \rho_{w}\right) \mathrm{cm} \\
h_{o}(z, 0)-z \rho_{o} / \rho_{w}=3.56\left(\rho_{o} / \rho_{w}\right) \mathrm{cm}
\end{gathered}
$$

where $h_{w}(z, t)$ and $h_{o}(z, t)$ are water and oil pressure heads expressed in water-equivalent heights, and $\rho_{w}$ and $\rho_{o}$ are water and oil mass densities $\left(\mathrm{g} \mathrm{cm}^{-1}\right)$, respectively. The initial condition corresponds to complete water saturation within the column since the oil-water capillary head, $h_{o w}=h_{o}-h_{w,}$ is equal to or less than zero for all depths. Note that the initial condition also implies that the total liquid saturation $S_{q}$ is equal to 1 , since the air-oil capillary head, $h_{s e}=h_{a}-h_{o}$, is equal to or less than zero for all depths (i.e., $S_{w}=S_{\imath}$ ). Lower boundary conditions $(z=52.5 \mathrm{~cm}$ ) were represented by specifying water heads and oil fluxes during the period $0<t<41.5$ min by

$$
\begin{gathered}
h_{w}(52.5, t)=27.3 \mathrm{~cm} \\
q_{o}(52.5, t)=0
\end{gathered}
$$

and for $t=41.5 \mathrm{~min}$ by

$$
\begin{gathered}
h_{w}(52.5, t)=1.5 \mathrm{~cm} \\
q_{0}(52.5, t)=0
\end{gathered}
$$

where $q_{o}(z, t)$ is the oil flux density. At the upper boundary $(z=0)$, a zero water flux was employed at all times and a falling oil head was imposed during imbibition of the oil slug followed by zero oil flux as

$$
\begin{gathered}
q_{w}(0, t)=0 \quad t>0 \\
h_{o}(0, t)=\left(\rho_{o} / \rho_{w}\right)\left[Q_{\text {tot }}-Q(t)\right] \quad Q(t)<Q_{\text {tot }} \\
q_{o}(0, t)=0 \quad Q(t) \geq Q_{\text {tot }}
\end{gathered}
$$

where

$$
Q(t)=\int_{0}^{t} q_{o}(0, t) d t
$$

and $Q_{\mathrm{tot}}=3.56 \mathrm{~cm}$ is the initial height of oil above the soil surface at $t=0$.

\section{Results AND Discussion}

\section{Experimental Results}

Measured water, oil, and total liquid saturations using the dual-energy gamma radiation apparatus for the five measurement locations are shown in Figures 2-6. Also shown are water and oil pressure heads expressed in centimeters of water height. As drainage of water from the bottom of the flow column was initiated, water saturation decreased at a very rapid rate near the upper surface (Figure 2) which was mirrored by an increase in oil saturation. All of the applied oil imbibed into the porous medium in $8.1 \mathrm{~min}$.

With the outflow level positioned at $z=25.2 \mathrm{~cm}$ not enough water drained from the column to allow air to infiltrate to $z=5.2 \mathrm{~cm}$ (Figure 2), i.e., measured total liquid saturations remain at unity. A rapid approach to equilibrium is evidenced by the small slope of saturation with respect to time following the initial rapid changes in liquid saturations. Measured cumulative water outflow volume from the column at $t=36.5 \mathrm{~min}$ was only $210 \mathrm{~mL}$, indicating only $10 \mathrm{~mL}$ of air had imbibed in the porous medium.

At $t=41.5 \mathrm{~min}$ the water table position was lowered to its final position at $z=51.0 \mathrm{~cm}$. As soon as the water table was lowered, there were rapid decreases in oil and water saturations (e.g., total liquid saturation) followed by very gradual decreases at later times. Shortly after lowering the water table to $z=51.0 \mathrm{~cm}$, air was present at $z=5.2 \mathrm{~cm}$. Changes in measured water and oil pressure heads at $z=5.2 \mathrm{~cm}$ also reflect lowering of the water table.

Only a small volume of oil penetrated to $15.2 \mathrm{~cm}$ below the soil surface (Figure 3) when the water table was positioned at $z=25.2 \mathrm{~cm}$. After the water table was lowered to $z=51.0 \mathrm{~cm}$, there were rapid decreases in water saturation and pressure 

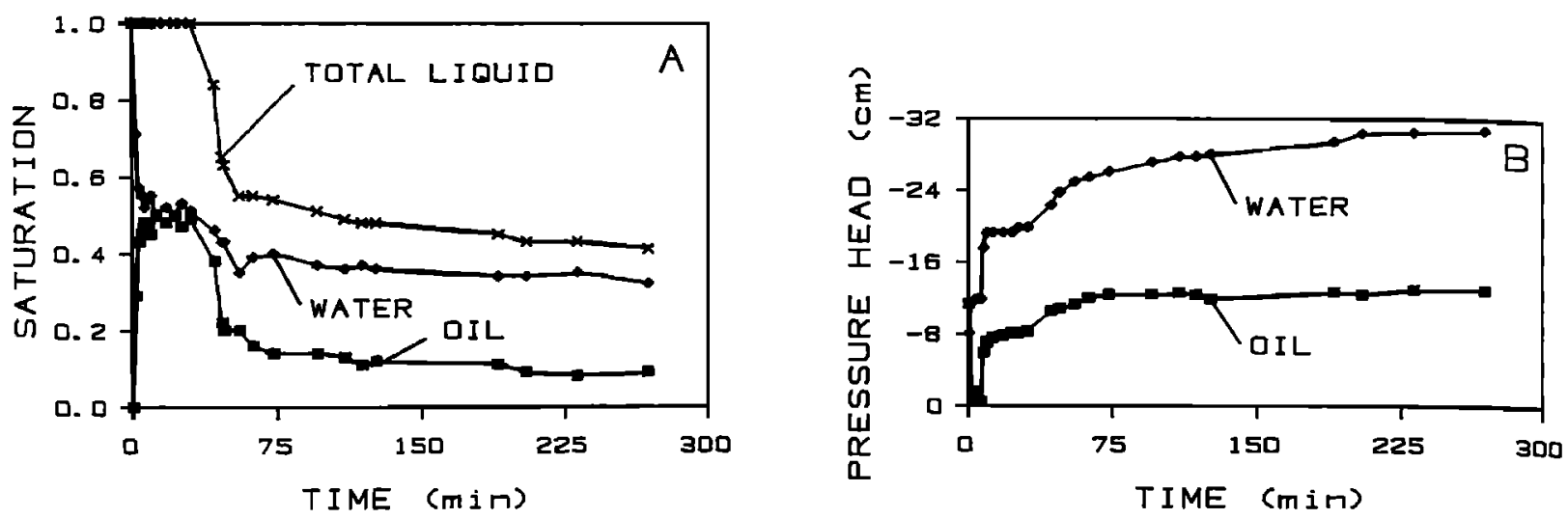

Fig. 2. Results of transient flow experiment at $5.2 \mathrm{~cm}$ depth showing (a) measured total liquid, water, and oil saturations and $(b)$ measured water and oil pressure heads as functions of time.

head with corresponding increases in oil saturation. Air did not penetrate to $z=15.2 \mathrm{~cm}$ until $85 \mathrm{~min}$ after commencement of drainage. At $z=25.2 \mathrm{~cm}$ (Figure 4), the elevation of the first water table position, oil was not detected until after the water table was lowered to $z=51.0 \mathrm{~cm}$. There was an anomaly in measured liquid saturations at about 50-60 min when it appeared that water saturation decreased without a corresponding increase in oil saturation which produced the dip in the total liquid saturation curve. At $t=65 \mathrm{~min}$, however, there was a substantial volume of oil in the porous medium at this depth. The anomaly may be a consequence of the rapidly changing liquid saturations relative to the duration of gamma counts. At later times water and oil saturations decreased gradually as equilibrium conditions corresponding to the final water table elevation were approached.

At $z=35.2 \mathrm{~cm}$ (Figure 5), which was $15.8 \mathrm{~cm}$ above the final water table position, the fluid system in the sandy porous medium changed from a single-fluid phase system (water) at early times to a two-fluid phase system (water and oil) at later times. Measured liquid saturations indicated that the porous medium at this depth remained essentially saturated with liquid for the duration of the experiment. When oil appeared at this depth at $t \cong 100 \mathrm{~min}$, there was a dramatic change in the oil pressure head (Figure 5). Treated (hydrophobic) ceramics are wet by organic liquids and air in preference to water; therefore a small volume of air may occur between the ceramic surface and the water phase when oil is not present.
Pressure readings from hydrophobic tensiometers in the absence of oil in the porous medium will reflect pressures of the water phase transferred by the gas film and modified due to air-water capillary effects in the porous medium and air-oil capillary effects at the tensiometer surface. When a continuous oil phase is present in the porous medium, oil will wet the hydrophobic ceramic suface in preference to air and oil pressure head measurements will reflect the pressure of the oil phase at that elevation. The conspicuous change in oil pressure head between $t=92$ to $100 \mathrm{~min}$ in Figure 5 corresponded to an oil saturation change from 0.01 to $0.05 \mathrm{~cm}^{3} \mathrm{~cm}^{-3}$. At the fifth measurement position, $z=45.2 \mathrm{~cm}$ (Figure 6), the porous medium remained essentially water saturated throughout the duration of the experiment. The large change in oil pressure head occurred when the measured oil saturation increased to approximately $0.02 \mathrm{~cm}^{3} \mathrm{~cm}^{-3}$.

Note that oil pressure heads at $z=15.2 \mathrm{~cm}$ (Figure 3) changed very little $(0.5 \mathrm{~cm})$ as the total liquid saturation decreased from 0.81 to 0.55 , whereas at $z=5.2 \mathrm{~cm}$ (Figure 2) the oil pressure heads changed from -10.5 to -12.4 as the total liquid saturation decreased from 0.84 to 0.54 and at $z=25.2$ $\mathrm{cm}$ (Figure 4) the oil pressure heads changed from -8.3 to -10.2 as the total liquid saturation decreased from 0.67 to 0.55 . This suggests that either the oil pressure transducer at $z=15.2 \mathrm{~cm}$ was not functioning properly at later times ( $>64$ $\mathrm{min}$ ) or that the porous medium was not packed tight enough against the chemically treated ceramic insert and when the
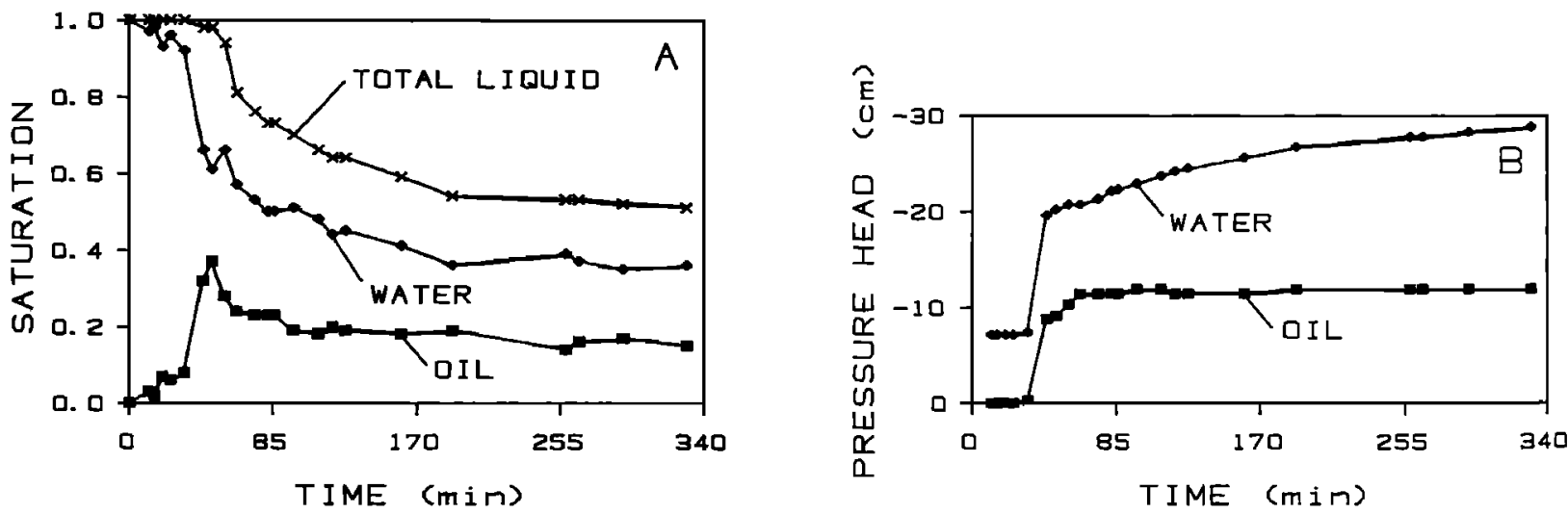

Fig. 3. Results of transient flow experiment at $15.2 \mathrm{~cm}$ depth showing (a) measured total liquid, water, and oil saturations and $(b)$ measured water and oil pressure heads as functions of time. 

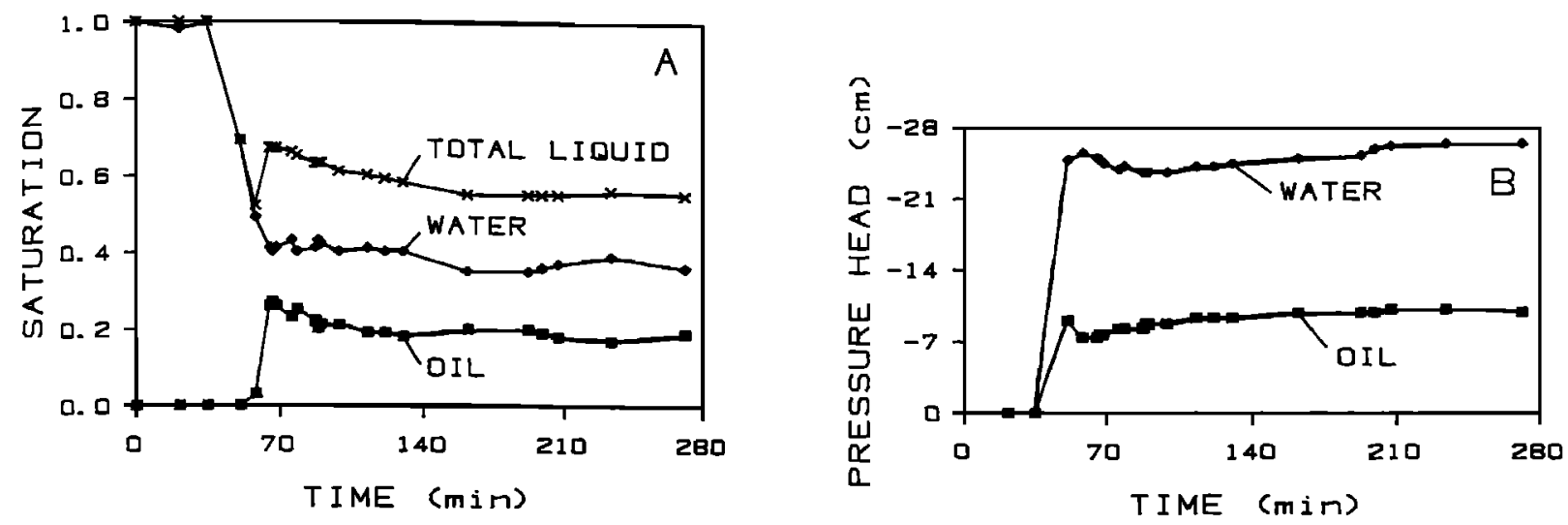

Fig. 4. Results of transient flow experiment at $25.2 \mathrm{~cm}$ depth showing (a) measured total liquid, water, and oil saturations and $(b)$ measured water and oil pressure heads as functions of time.

total liquid saturation decreased below approximately 0.8 contact between the treated ceramic surface and the liquids contained in the porous medium was severed as liquid drained from the relatively large flow channel between the porous medium and treated ceramic tensiometer. Consequently, we would expect the oil pressure heads to be slightly more negative at later times than those measured at $z=15.2 \mathrm{~cm}$.

\section{Model Validation}

Calibration of the three-phase $k-S-P$ model was achieved either by use of two-phase air-water $S-P$ data in conjunction with interfacial tension measurements (method 1) or by directly fitting parameters to three-phase $S-P$ data obtained from the transient flow experiment (method 2). To evaluate the accuracy of these procedures, three-phase $S_{w}\left(h_{\text {ow }}\right)$ and $S_{t}\left(h_{a 0}\right)$ data, obtained from oil and water saturation and pressure measurements during the transient flow experiment, are compared in Figure 7 with predictions by the two methods. Differences in $S_{w}\left(h_{o w}\right)$ predictions for the two methods are minor and both agree well with the observed data. Scatter in the data is fairly substantial, reflecting composite effects of measurement errors and heterogeneity in packing from depth to depth.

Discrepancies between observed and predicted $S_{t}\left(h_{a o}\right)$ relations are much greater than for $S_{w}\left(h_{o w}\right)$ results. The scaling factor estimated from interfacial tension data (i.e., method 1 ) is significantly greater than that inferred directly from the three- phase data (method 2). Scatter about the method 2-fitted curve is more severe than for the $S_{w}\left(h_{\text {ow }}\right)$ data. Since deviations between observed and fitted $S_{t}\left(h_{a 0}\right)$ and $S_{w}\left(h_{o w}\right)$ for the same observation depths were not significantly correlated, porous medium heterogeneity seems an unlikely cause of the large scatter for $S_{s}\left(h_{a 0}\right)$ data. Since errors in oil pressures would effect $S_{w}\left(h_{o w}\right)$ as well as $S_{r}\left(h_{a 0}\right)$ results, this source of error is also improbable. Inspection of the $S_{\mathrm{r}}\left(h_{a 0}\right)$ data points which lie above the method 2-fitted curve indicates that these values largely correspond to measurements at the $15.2-\mathrm{cm}$ depth (diamonds) where pressure readings remained nearly constant as the total liquid saturation decreased from 0.81 to 0.55 . If the latter points are ignored, the agreement between method 1 and method $2 S_{\mathrm{s}}\left(h_{a 0}\right)$ curves would be substantially improved. In another study [Lenhard and Parker, 1988], we have found good agreement between static $S_{r}\left(h_{a 0}\right)$ relations in three-fluid phase systems and between static two-phase air-oil and scaled static two-phase air-water $S-P$ measurements. Although Figure 7 shows transient $S-P$ relations, they should not deviate significantly from static $S-P$ relations [Topp et al., 1967; Corey and Brooks, 1975; Perroux et al., 1982].

Another potential source of error, particularly at lower elevations, is the assumption of constant air pressure. Air pressure at its deepest penetration into a porous medium can be expected to fluctuate in an erratic manner (A. T. Corey, personal communication, 1987). Because of the coarse nature of the porous medium, variations in gas pressure of only a few
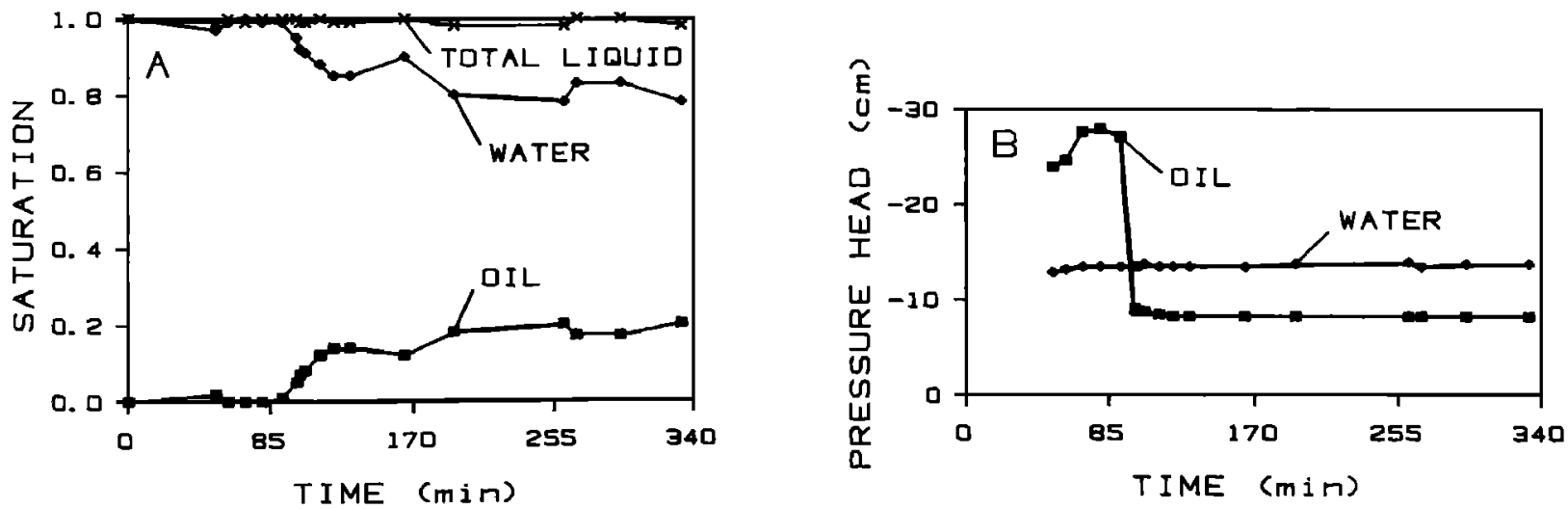

Fig. 5. Results of transient flow experiment at $35.2 \mathrm{~cm}$ depth showing (a) measured total liquid, water, and oil saturations and $(b)$ measured water and oil pressure heads as functions of time. 

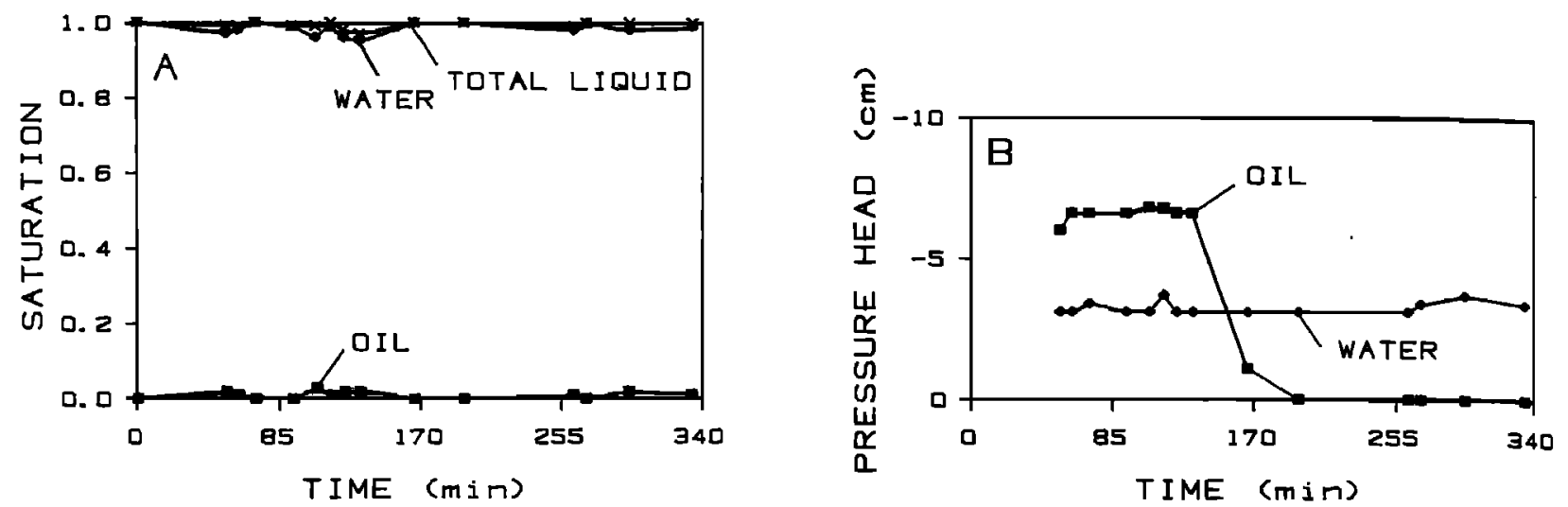

Fig. 6. Results of transient flow experiment at $45.2 \mathrm{~cm}$ depth showing (a) measured total liquid, water, and oil saturations and $(b)$ measured water and oil pressure heads as functions of time.

$\mathrm{cm}$ water, due to barometric pressure fluctuations or flow induced gradients, could effect very large changes in fluid saturations (see Figure 7). Unfortunately, since gas pressures were not measured, definitive resolution of the source of the apparent discrepancies in the present experiment cannot be ascertained.

Fluid saturations and liquid pressures were simulated with the multiphase flow code for the period in which measurements were conducted using method 1 and method $2 k-S-P$ model parameters and results are shown in Figures 8-11. Solid curves represent predicted saturations and pressure heads obtained when the model was calibrated by method 1 and dashed curves when the model was calibrated by method 2. Figure 8 shows comparisons between predicted and measured water and oil saturations and pressure heads as a function of time for the uppermost measurement position $(z=5.2$ $\mathrm{cm})$. The initial rapid rate of water drainage and oil infiltration was predicted by both method 1 and method 2 model parameters. The predicted time required for all of the oil to infiltrate into the porous medium for method 1 parameters was 9.9 min and for method 2 parameters it was $11.8 \mathrm{~min}$, which correspond favorably to the observed time of $8.1 \mathrm{~min}$. Agreement between predicted and measured oil saturations was quite good. Although agreement between predicted and measured water saturations was not as good, the simulations predicted the initial rapid water drainage, the small rate of water saturation change immediately prior to lowering the water table to $z=51 \mathrm{~cm}$ and the gradual decrease in water saturations at latter times. There was also good agreement between simulated and observed water and oil pressure heads at early times.

Figure 9 shows similar comparisons for the 15.2-cm depth. Predicted water and oil saturations and pressure heads compared favorably to those measured except that the oil front arrived earlier and more water drained initially. At $z=25.2$ $\mathrm{cm}$ (Figure 10) correspondence between simulated and mea-
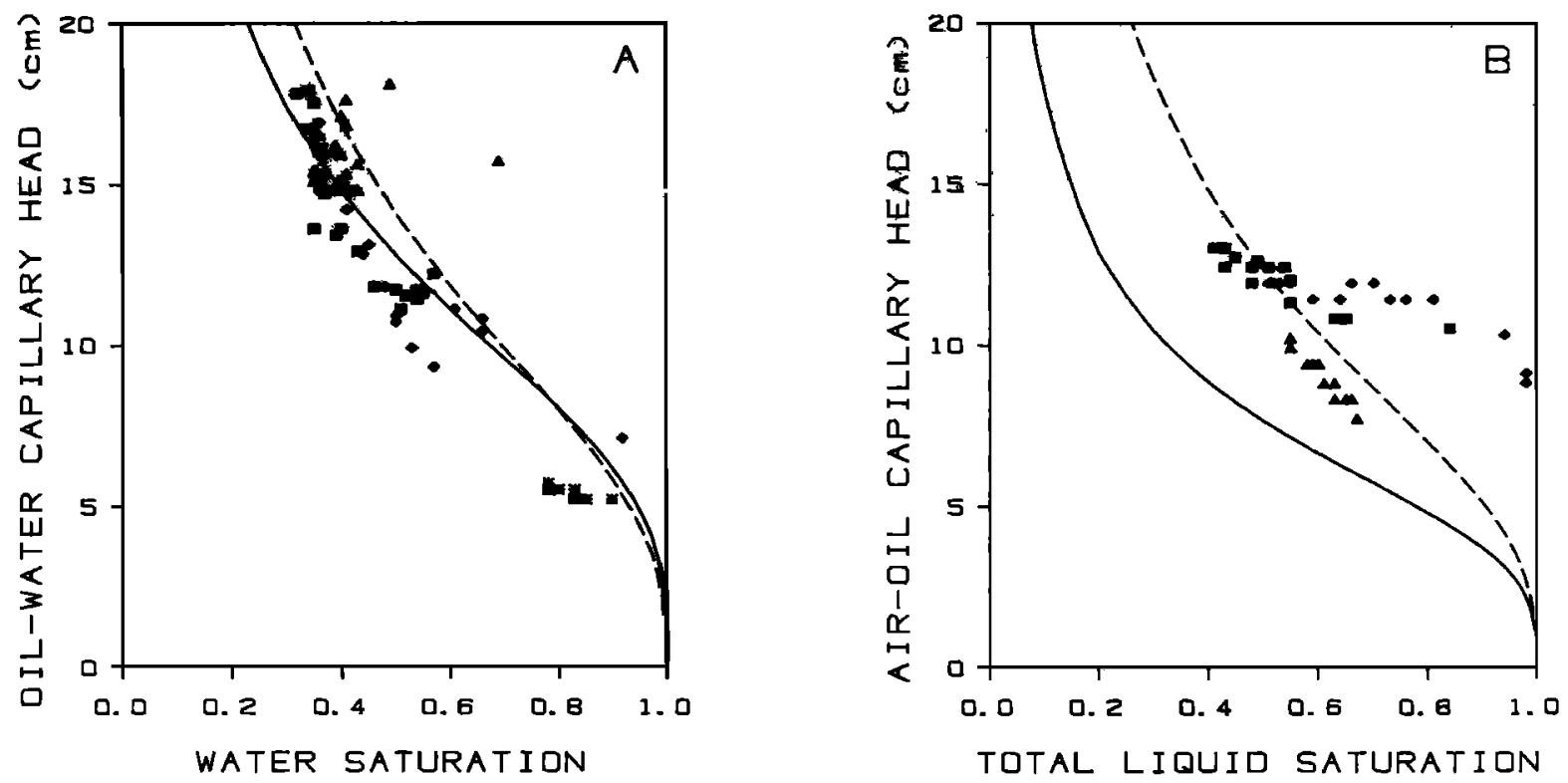

Fig. 7. Comparison of observed saturation-pressure data in transient three-phase flow experiment (points) and predictions using method 1 parameters (solid curves) and method 2 (dashed curves): (a) water saturation versus oil-water capillary head and $(b)$ total liquid saturation versus air-oil capillary head. Squares show measurements at the 5.2-cm depth, diamonds show the 15.2-cm depth, triangulars show the 25.2-cm depth, and asterisks show the 35.2-cm depth. 

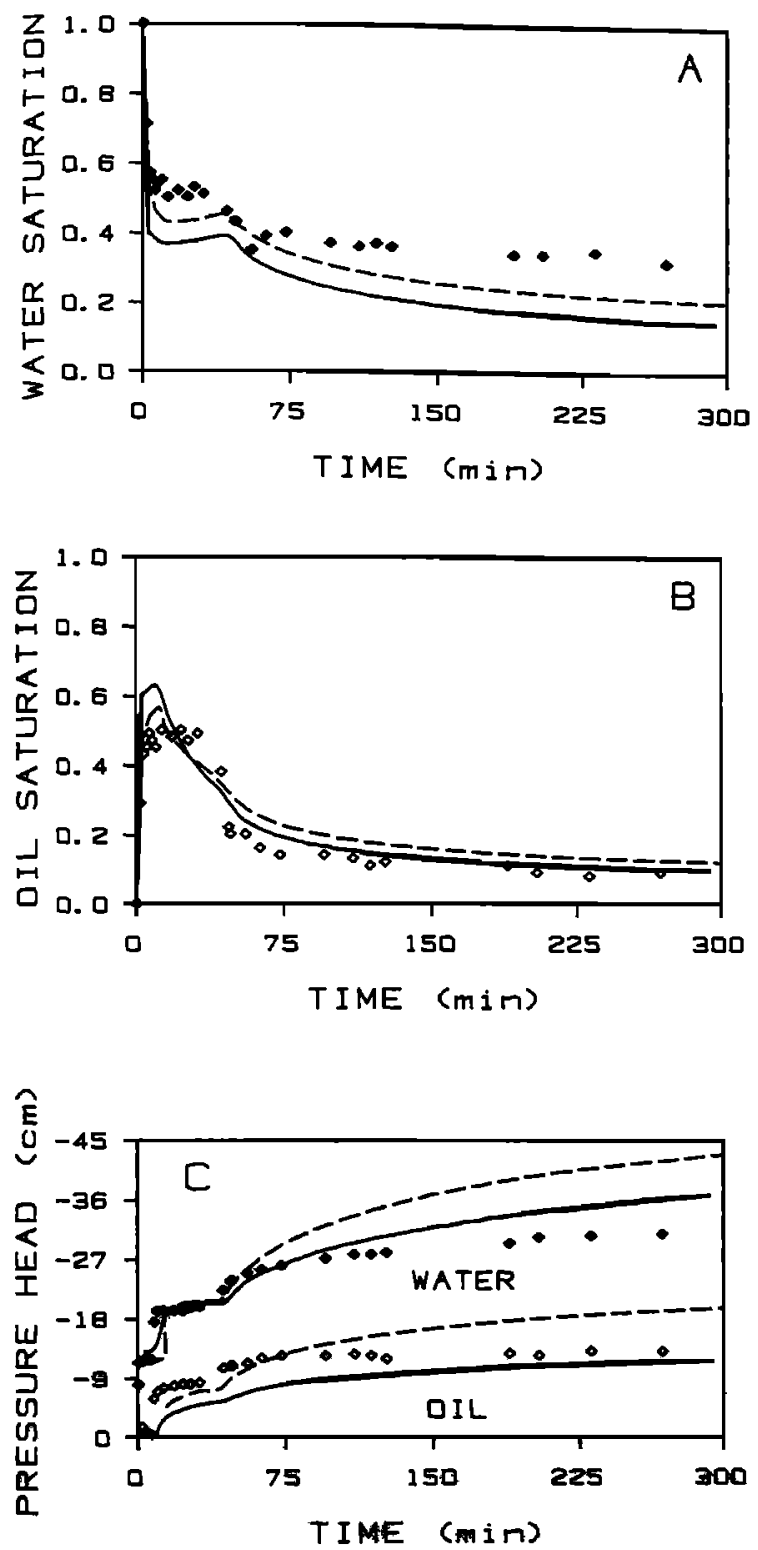

Fig. 8. Comparison of measured water saturations and heads (solid symbols) and oil saturations and heads (open symbols) with numerically simulated values at $5.2 \mathrm{~cm}$ depth using model parameters from method 1 (solid curves) and method 2 (dashed curves): (a) measured versus predicted water saturations, $(b)$ measured versus predicted oil saturations, and $(c)$ measured versus predicted water and oil pressure heads.

sured saturations and pressure heads was generally very good. However, as with the $15.2 \mathrm{~cm}$ depth, oil was predicted to appear earlier than was observed.

Figure 11 shows comparisons between predicted and measured saturations and pressure heads as a function of time for $z=35.2 \mathrm{~cm}$. Observed water saturations and pressure heads were poorly predicted by both method 1 and method 2 model parameters. Whereas measured liquid saturations indicated that the porous medium remained essentially liquid saturated at this depth, the simulations predict a three-phase air-oilwater system at later times with a total liquid saturation of approximately 0.8 . At $z=45.2 \mathrm{~cm}$, predicted saturations and pressure heads also compared poorly with measured values.
Both method 1 and method 2 model parameters predicted a two-phase oil-water fluid system at $z=45.2 \mathrm{~cm}$ with an oil saturation of approximately 0.2 at later times, while measured saturations indicated the porous medium remained essentially water saturated (not illustrated).

The increasing discrepancy between observed and predicted behavior at greater depths may be related to the anomolous behavior of transient-measured $S_{t}\left(h_{o p}\right)$ data (Figure $7 b$ ) which indicated lower liquid saturations at a given capillary head than those predicted from air-water data via method 1 or even than those fitted by method 2. Deviations from the assumption of constant gas phase pressure were suggested as a possible explanation for this behavior earlier. Since deviations of gas pressures from atmospheric should increase with depth under the conditions of this experiment, poorer correspon-
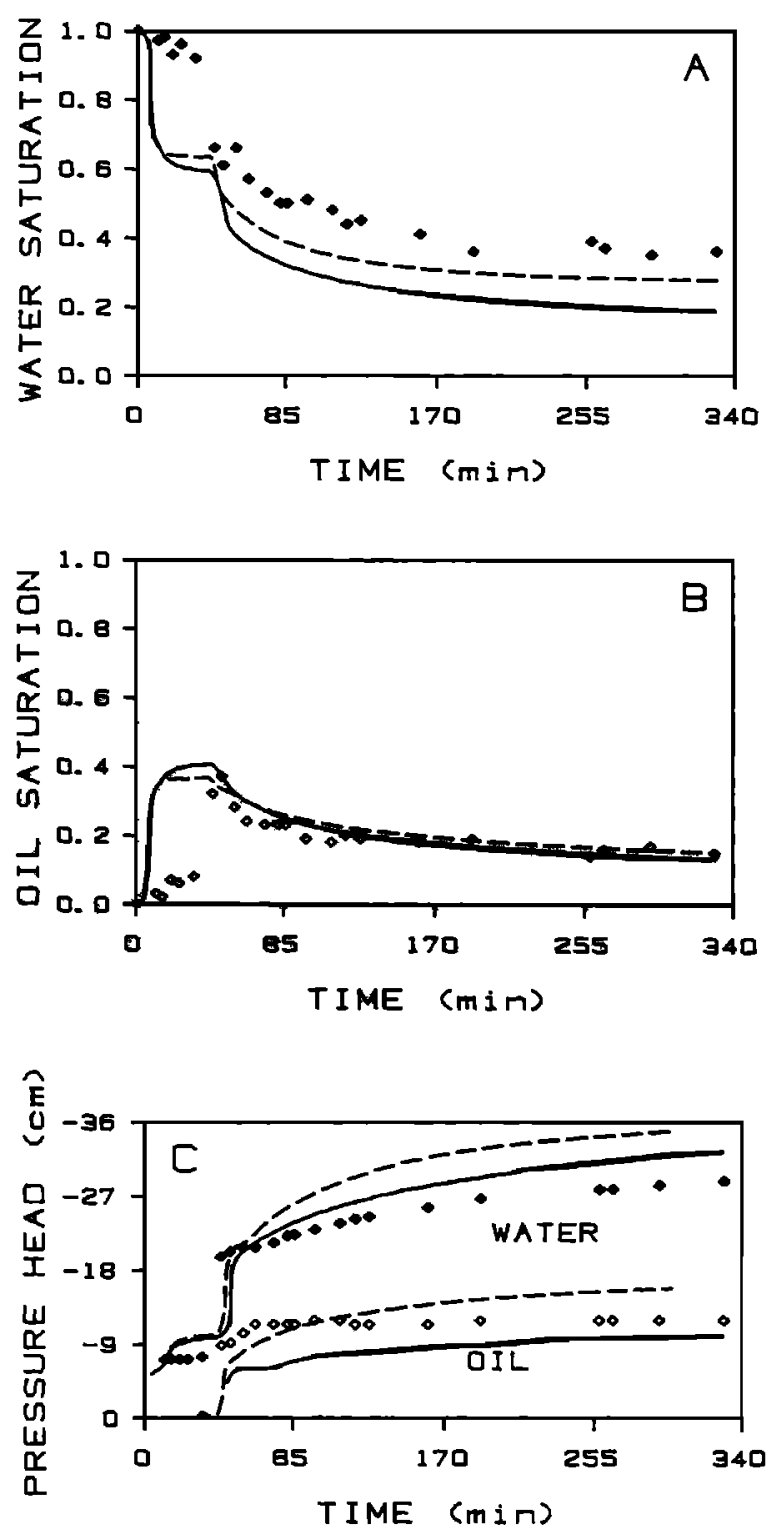

Fig. 9. Comparison of $(a)$ measured versus predicted water saturation, $(b)$ measured versus predicted oil saturation. and (c) measured versus predicted water and oil pressure heads at $15.2 \mathrm{~cm}$ depth. Symbols and curves as in Figure 8. 

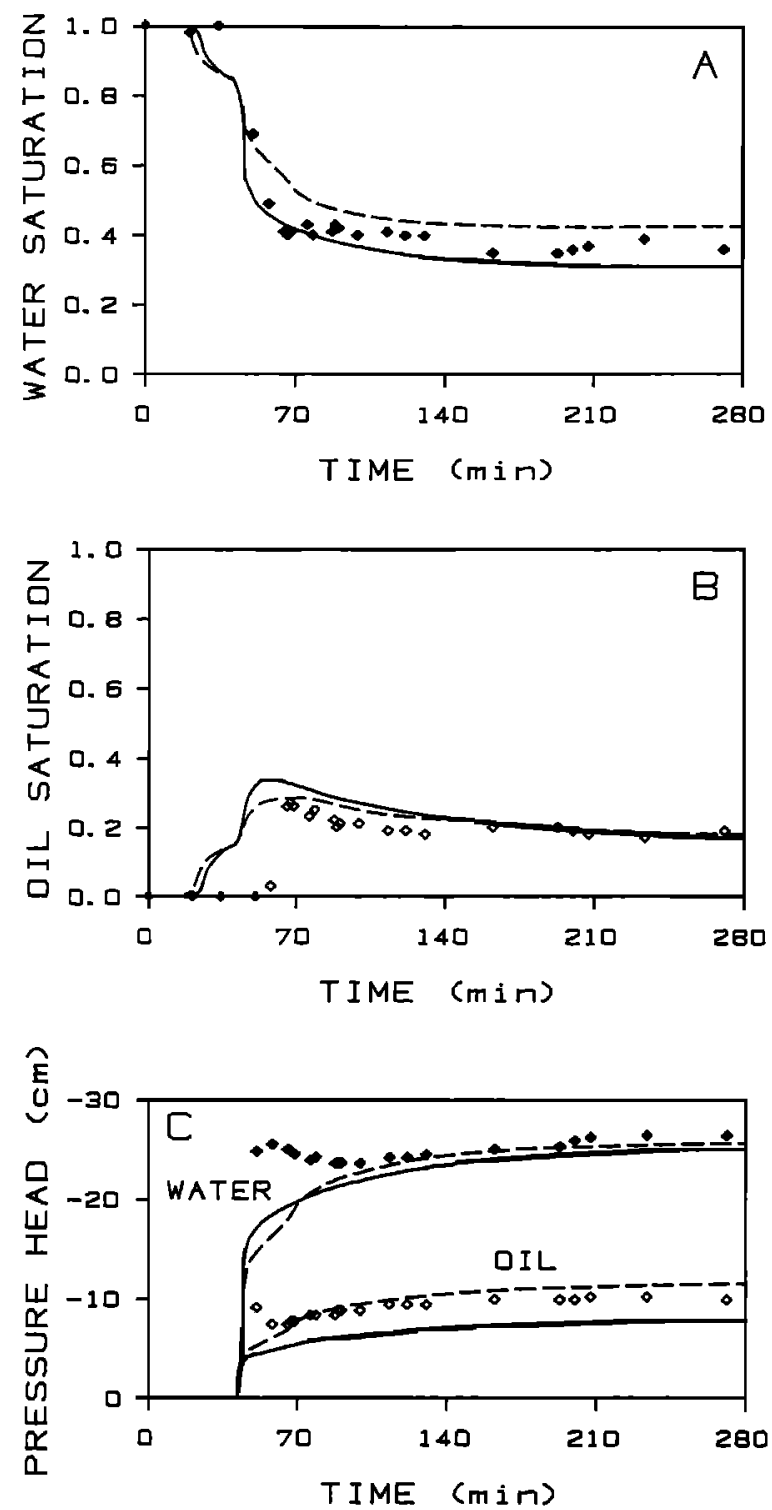

Fig. 10. Comparison of $(a)$ measured versus predicted water saturation, (b) measured versus predicted oil saturation, and (c) measured versus predicted water and oil pressure heads at $25.2 \mathrm{~cm}$ depth. $S y \mathrm{~m}$ bols and curves as in Figure 8.

dence with predictions would result at greater depths. Other anamolies in the predicted behavior could arguably be blamed on gas phase effects as well. For example, the slower oil drainage over that predicted (Figures $8 b$ and $9 b$ ) and generally higher observed water saturations (Figures $(8 a$ and $9 a$ ) and less negative liquid pressures (Figures $8 c$ and $9 c$ ) could reflect appreciable impedance to gas phase flow.

Alternatively, it is possible that the $k-S-P$ constitutive model fails to describe fluid behavior accurately in the region of low capillary pressures. The van Genuchten [1980] $S-P$ model, for example, imposes no finite fluid entry value corresponding to the largest interconnected network of pore sizes with the porous medium, thus yielding a continuous $S-P$ model for all positive capillary pressures. In contrast, the Brooks and Corey [1966] model assumes a finite fluid entry value which, in turn, infers somewhat different fluid behavior. Whether such differences in the form of the constitutive model would greatly affect the numerical results is difficult to assess a priori, but future attention to the possibility seems warranted.

\section{Summary AND CONCLUSIONS}

A three-phase air-oil-water flow experiment was designed and conducted with simultaneous measurements of liquid saturations and pressures during monotonic water and total liquid drainage. Oil and water saturations were measured with a dual-energy gamma radiation apparatus after doping the liquid phases to enhance separation of gamma attenuation coefficients. Oil and water pressures were measured with hydrophobic and hydrophilic ceramic teniometers, respectively.

Measured water saturation versus oil-water capillary head data during the three-phase flow experiment agreed well with predictions using the model of Parker et al. [1987] calibrated from static air-water $S-P$ relations and interfacial tension data (method 1). Measured total liquid saturation versus air-oil capillary head data, computed under the assumption of uniform zero gas pressure, deviated more severely from the air-water data predictions and exhibited marked scatter. If was inferred that these discrepancies were likely due to deviations from the
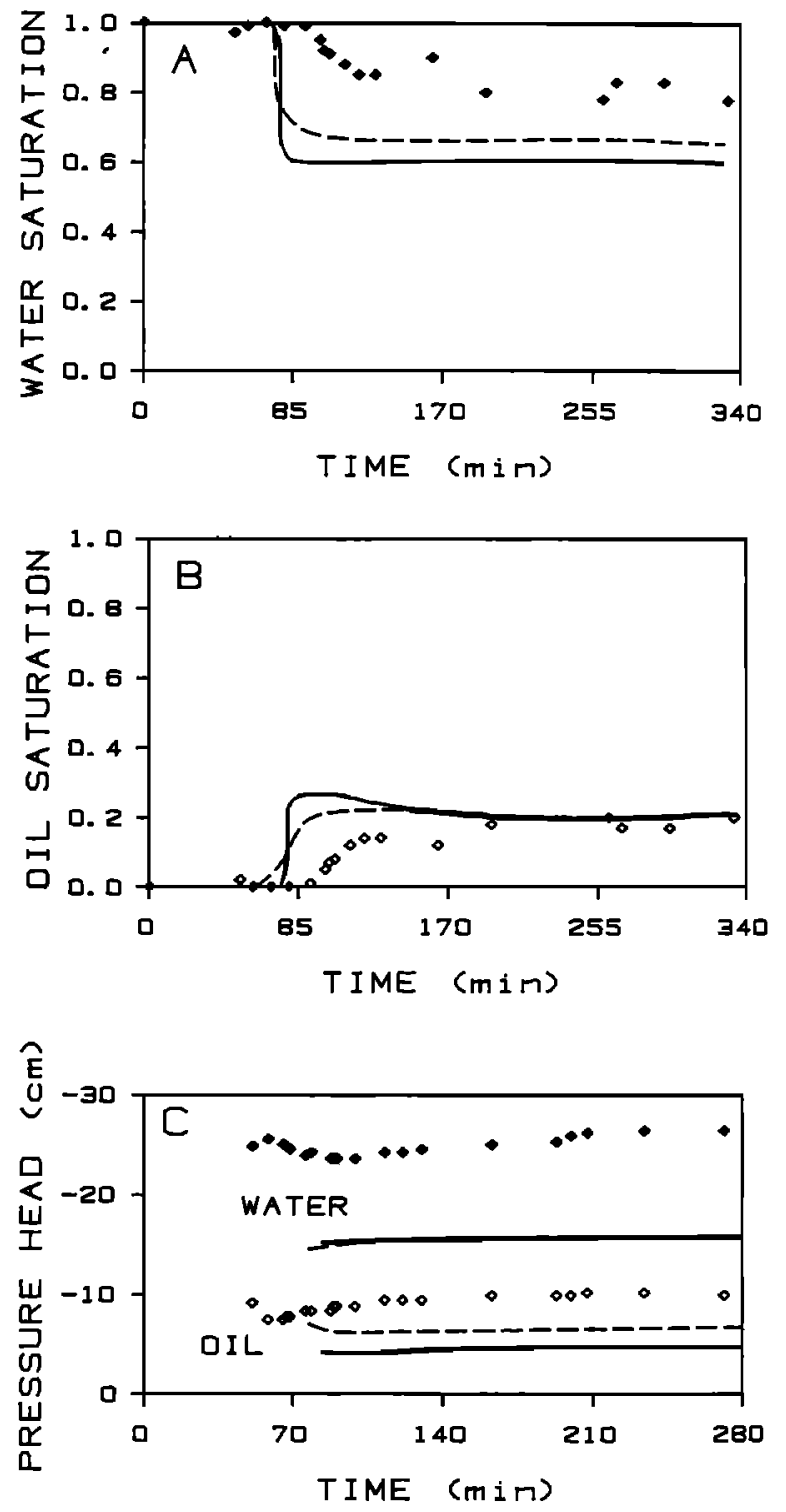

Fig. 11. Comparison of (a) measured versus predicted water saturation, $(b)$ measured versus predicted oil saturation, and $(c)$ measured versus predicted water and oil pressure heads at $35.2 \mathrm{~cm}$ depth. Symbols and curves as in Figure 8. 
assumption of constant gas phase pressure. Model parameters were also evaluated by directly fitting to the observed threefluid phase $S-P$ data (method 2).

Generally, good agreement was found between measured water and oil saturations and pressure histories at most measurement locations during the transient flow experiment and those predicted by a finite element multiphase flow code using the $k-S-P$ model of Parker et al. [1987]. Sensitivity of numerical results to calibration method were rather mild such that little if any advantage accrued from use of the much more data intensive method 2 . Thus the simpler method 1 appears to represent a practical and valuable calibration method for routine usage.

At the two lower measurement elevations, experimental and simulated saturations and pressures agreed less well. Deviations from the assumption of negligible gas pressure gradients was again suggested as a possible cause of these discrepancies; however, inaccuracies in the constitutive model in the region of low capillary pressures was not ruled out as a possible contributor to the errors. Further attention to the problem of gas phase convection and to alternate forms for the constitutive relations is clearly warranted.

Acknowledgments. This work was supported by the U.S. Environmental Protection Agency (USEPA) through the R. S. Kerr Environmental Research Laboratory under assistance agreement CR-812073. The paper has not been subjected to the USEPA's peer and administrative review and therefore does not necessarily reflect the views of the Agency. Special appreciation is due to Marshall McCord for invaluable assistance in conducting the experiments.

\section{REFERENCES}

Abriola, L. M., and G. F. Pinder, A multiphase approach to the modeling of porous media contamination by organic compounds, 2, Numerical simulation, Water Resour. Res., 21, 19-26, 1985.

Adamson, A. W., Physical Chemistry of Surfaces, 2nd ed., WileyInterscience, New York, 1967.

Aziz, K., and A. Settari, Petroleum Reservoir Simulation, Applied Science Publishers, London, 1979.

Baehr, A. L., and M. Y. Corapcioglu, A compositional multiphase model for groundwater contamination by petroleum products, 2 , Numerical solution, Water Resour. Res., 23, 201-213, 1987.

Brooks, R. H., and A. T. Corey, Properties of porous media affecting fluid flow, J. Irrig. Drain. Div. Am. Soc. Civ. Eng., 92, 61-68, 1966.

Corey, A. T., and R. H. Brooks, Drainage characteristics of soils, Soil Sci. Soc. Am. J., 39, 251-255, 1975.

Corey, A. T., C. H. Rathjens, J. H. Henderson, and M. R. J. Wyllie, Three-phase relative permeability, Trans. AIME, 207, 349-351, 1956.

Eckberg, D., and D. K. Sunada, Nonsteady three-phase immisicible fluid distribution in porous media, Water Resour. Res., 20, 18911897, 1984.

Faust, C. R., Transport of immiscible fluids within and below the unsaturated zone: A numerical model, Water Resour. Res., 21, 587$596,1985$.

Ferrand, L. A., P. C. D. Milly, and G. F. Pinder, Dual-gamma attenuation for the determination of porous medium saturation with respect to three fluids, Water Resour. Res., 22, 1657-1663, 1986.

Fritton, D. D., Resolving time, mass absorption coefficient and water content with gamma-ray attenuation, Soil Sci. Soc. Am. J., 33, 651$655,1969$.

Hockmuth, D. P., and D. K. Sunada, Groundwater model of twophase immiscible flow in coarse material, Ground Water, 23, 617$626,1985$.

Honarpour, M., L. Koederitz, and A. H. Harvey, Relative Permeability of Petroleum Reservoirs, CRC Press, Boca Raton, Fla. 1986.

Hopmans, J. W., and J. H. Dane, Calibration and use of a dualenergy gamma system, pressure transducers, and thermocouples to determine volumetric water content, dry bulk density, soil water pressure head, and temperature in soil columns, Agron and Soils Dep. Ser. Rep. 101, Ala. Agric. Exp. Stat., Auburn Univ., 1985.

Hopmans, J. W., and J. H. Dane, Calibration of a dual-energy gamma radiation system for multiple point measurements in a soil, Water Resour. Res. 7, 1009-1114, 1986.

Kuppusamy, T., J. Sheng, J. C. Parker, and R. J. Lenhard, Finite element analysis of multiphase immiscible flow through soils, Water Resour. Res., 23, 625-631, 1987.

Lenhard, R. J., and J. C. Parker, Measurement and prediction of saturation-pressure relationships in three-phase porous media systems, J. Contam. Hydrol., 1, 407-424, 1987 a.

Lenhard, R. J., and J. C. Parker, A model for hysteretic constitutive relations governing multiphase flow, 2, Permeability-saturation relations, Water Resour. Res. 23, 2197-2206, $1987 b$.

Lenhard, R. J., and J. C. Parker, Experimental validation of the theory of extending two-phase saturation-pressure relations to three-fluid phase systems for monotonic drainage paths, Water Resour. Res., 24, 373-380, 1988.

Leverett, M. C., Capillary behavior in porous solids, Trans. AIME, $142,152-169,1941$.

Leverett, M. C., and W. B. Lewis, Steady flow of gas-oil-water mixtures through unconsolidated sands, Trans. AIME, 142, 107-116, 1941.

Nolziger, D. L., and D. Swartzendruber, Material content of binary physical mixtures as measured with a dual-energy beam of gamma rays, J. Appl. Phys., 45, 5443-5449, 1974.

Parker, J. C., and R. J. Lenhard, A model for hysteretic constitutive relations governing multiphase flow, 1 , Saturation-pressure relations, Water Resour. Res., 23, 2187-2196, 1987.

Parker, J. C., R. J. Lenhard, and T. Kuppusamy, A parametric model for constitutive properties governing multiphase flow in porous media, Water Resour. Res., 23, 618-624, 1987.

Perroux, K. M., P. A. C. Raats, and D. E. Smiles, Wetting moisture characteristic curves derived from constant-rate infiltration into thin soil samples, Soil Sci. Soc. Am. J., 46, 231-234, 1982.

Oak, M. J., and R. Ehrlich, A new X-ray absorption method for measurement of three-phase relative permeability, Rep. SPE 14420 , Soc. of Pet. Eng. Dallas, Tex, 1985.

Ogden, M. W., Deactivation and preparation of fused silica open tubular columns for gas and supercritical fluid chromatography, Ph.D. thesis, Va. Polytechnic Inst. and State Univ., Blacksburg. 1985.

Osborne, M., and J. F. Sykes, Numerical modeling of immiscible organic transport at the Hyde Park landfill, Water Resour. Res., 22, 25-33, 1986.

Saraf, D. N., and I. Fatt, Three-phase relative permeability measurement using a nuclear magnetic resonance technique for extimating fluid saturation, Soc. Pet. Eng. J., 9, 235-242, 1967.

Schiegg, H. O., Fundamentals, setups and results of laboratory experiments on oil propagation in aquifers, Rep. $V A W-43$, Versuchsanstalt fur Wasserbau, Zurich, 1980.

Schiegg, H. O., and J. F. MeBride, Laboratory setup to study twodimensional multiphase flow in porous media, in Proceedings, Petroleum Hydrocarbons and Organic Chemicals in Ground Water Conference, National Water Well Association, Worthington, Ohio, 1988.

Snell, R. W., Three-phase relative permeability in an unconsolidated sand, J. Inst. Pet., 48, 80-88, 1962.

Topp, G. C., A. Klute, and D. B. Peters, Comparison of water content-pressure head data obtained by equilibrium, steady-state, and unsteady-state methods, Soil Sci. Soc. Am. J., 3I, 312-314, 1967.

Van Genuchten, M. T., A closed-form equation for predicting the hydraulic conductivity of unsaturated soils, Soil Sci. Soc. Am. J., 44, $892-898,1980$.

J. H. Dane, Department of Agronomy and Soils, Auburn University, Auburn, AL 36849.

J. J. Kaluarachchi, R. J. Lenhard, and J. C. Parker, Center for Environmental and Hazardous Materials Studies, 332 Smyth Hall, Virginia Polytechnic Institute and State University, Blacksburg, VA 24061 .

(Received May 28, 1987; accepted February 11, 1988; revised February 16, 1988.) 